La tension narrative dans le roman policier contemporain à travers Sept ans après... de Guillaume Musso
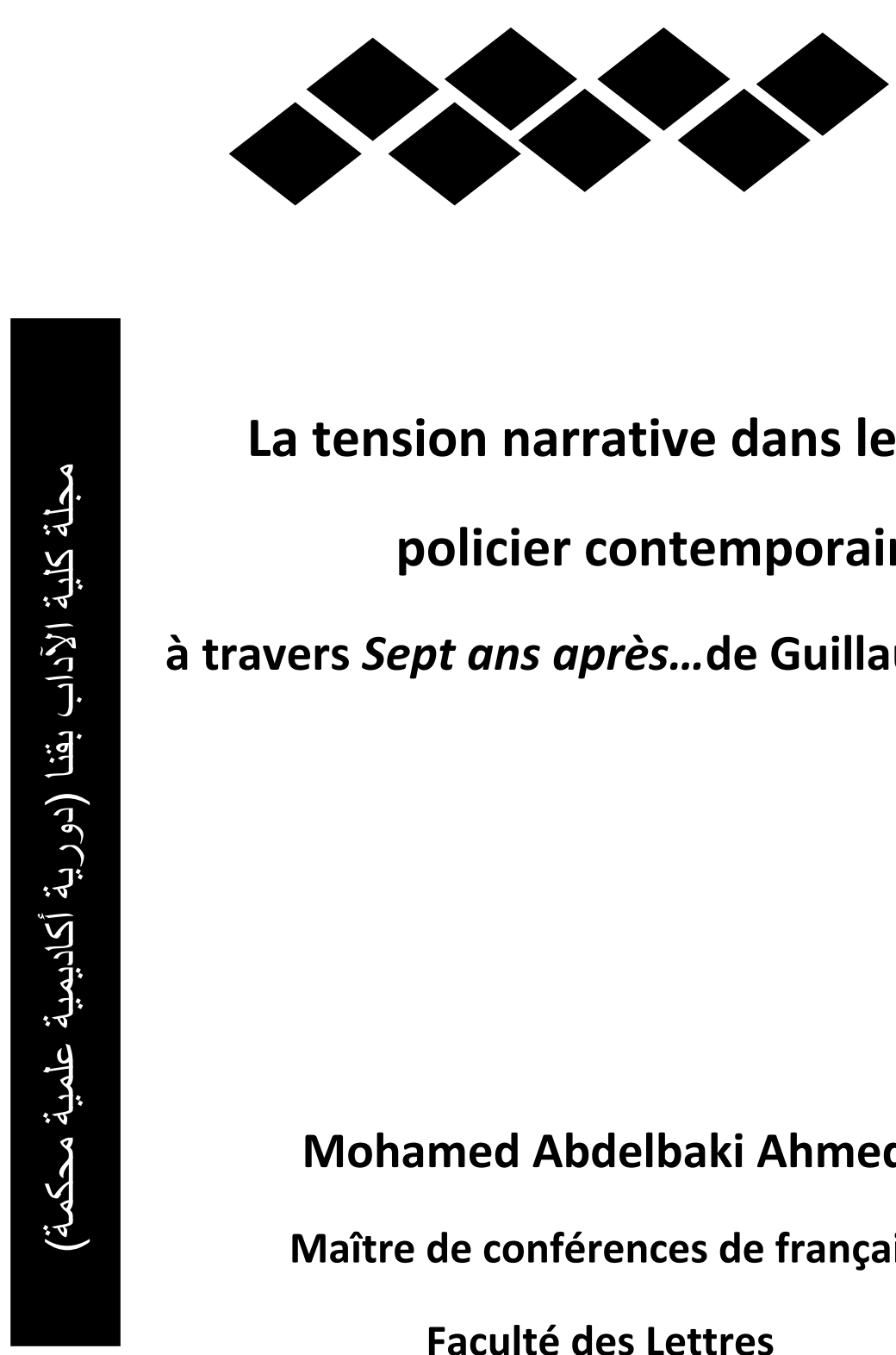

La tension narrative dans le roman policier contemporain

à travers Sept ans après...de Guillaume Musso

\author{
Mohamed Abdelbaki Ahmed \\ Maître de conférences de français
}

Faculté des Lettres 
La tension narrative dans le roman policier contemporain à travers Sept ans après... de Guillaume Musso

التثويق السردى فى الرواية البوليسية المعاصرة من خلال رواية بعد سبع سنوات... للكاتب الفرنسى جييوم موسو

$$
\begin{aligned}
& \text { د. محمد عبد الباقى أحمد } \\
& \text { مدرس اللغة الفرنسية } \\
& \text { كلية الاداب بقنا } \\
& \text { جامعة جنوب الوادى الادى }
\end{aligned}
$$

تتناول هذه الدراسة التشويق السردي في رواية " بعد سبع سنوات" للكاتب

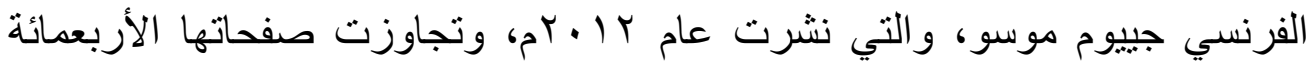

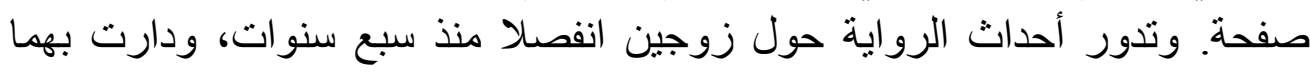

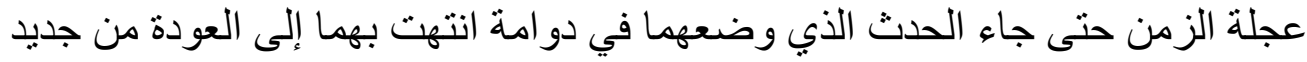

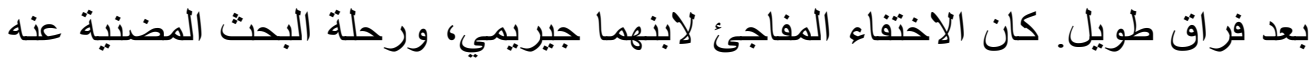

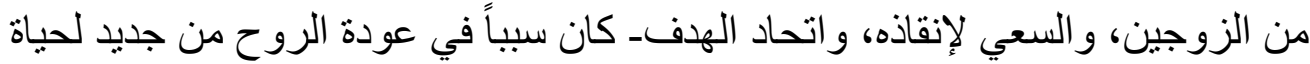
زوجية كانت قد قضت.وقد أبدع المؤلف؛ حيث كان لهذه الأحداث التهاث المثيرة أثراً بالغاً

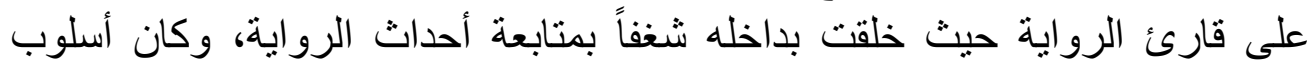

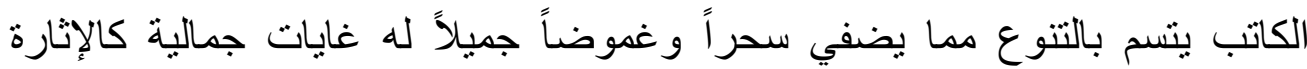
الذهنية و التثويق العقلي ولفت الانتباه.

وتمثل الهذف من البحث في التعرف على الأدوات والأساليب التي وظفها

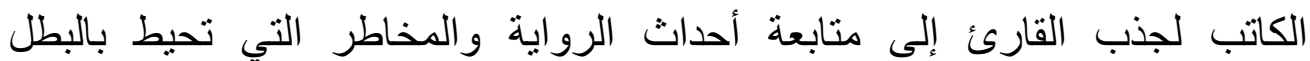

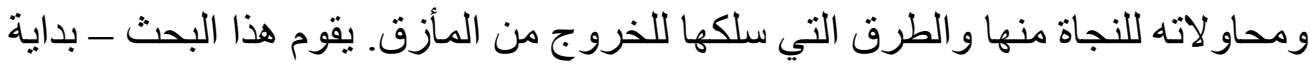

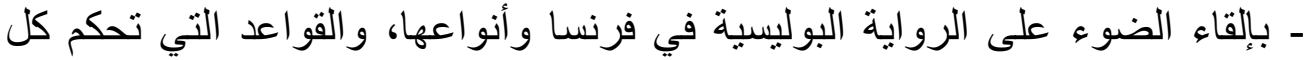

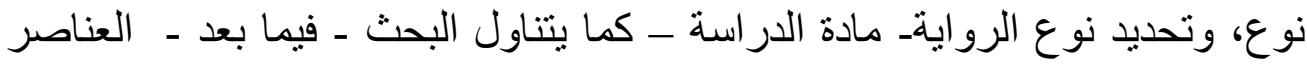

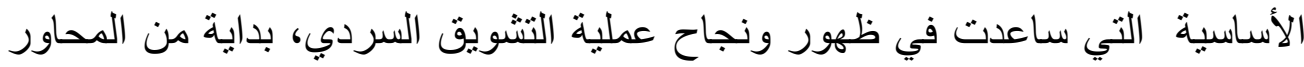

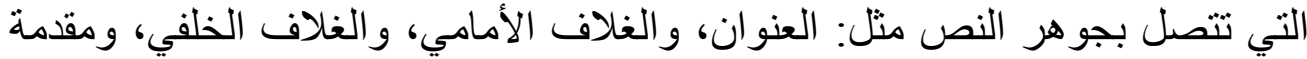

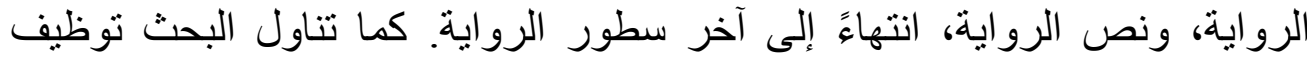

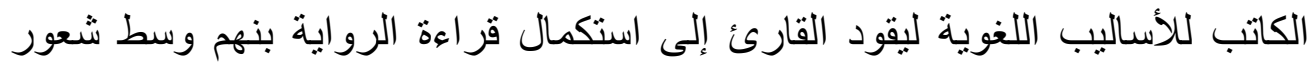

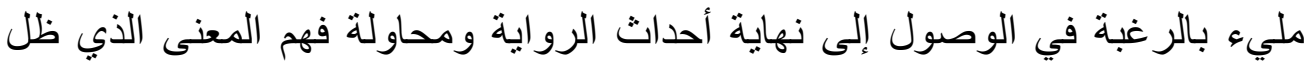
يدور في نفس الكاتب. 


\title{
La tension narrative dans le roman policier contemporain à travers Sept ans après... de Guillaume Musso
}

\author{
Mohamed Abdelbaki Ahmed \\ Maître de conférences de français \\ Faculté des Lettres \\ Université du Sud de la Vallée
}

\section{Introduction}

Dans cette recherche, nous étudierons la tension narrative dans Sept ans après de Musso, un roman écrit en 2012 qui raconte l'histoire d'un couple séparé qui a deux enfants. L'axe de ce roman est basé sur la disparition soudaine de leur fils Jeremy et comment tous les deux ont fait tout le possible pour le retrouver. Le lecteur du roman reste toujours vigilant à la lecture du roman puisque l'auteur utilise une tension narrative qui le tient jusqu'aux dernières lignes du roman. Notre objectif c'est de mettre l'accent sur cette technique narrative utilisée par l'auteur à l'égard de l'action du roman et de traiter les moyens narratifs que l'auteur a employés pour convaincre le lecteur à continuer et à attendre impatiemment la fin du récit. Nous traiterons d'abord les genres du roman policier et ensuite nous essayerons de délimiter d'après quel genre policier appartient le roman étudié. Nous analyserons ensuite les éléments qui ont déjà aidé à la production de la tension narrative à partir du paratexte jusqu'à la fin du texte et nous traiterons également les autres procédés stylistiques qui ont déjà construit cette tension narrative chez le lecteur potentiel du récit.

\section{1 - Le roman policier}

Le roman policier regroupe une multitude de formes différentes: le roman noir, le roman à énigme, et le roman à suspense. Le genre policier est formé d'emprunts aux autres genres littéraires mais peu à peu il devient un genre autonome avec des règles spécifiques. Le roman policier représente le récit rationnel d'une enquête menée sur un problème dont le ressort principal est un crime $^{1}$ ou à vrai dire il 

après... de Guillaume Musso

représente la trace romanesque d'une quête dont le but est de rétablir un équilibre qui a été rompu après une transgression sociale ${ }^{2}$. Les critiques littéraires classent le genre policier dans la catégorie de la paralittérature et les lecteurs du genre policier font d'Edgar Poe le père et le fondateur du genre ${ }^{3}$ tandis que Marc Lits dans son livre L'énigme criminelle considère le roman feuilleton du XIXe siècle comme origine du genre policier. Dès la fin du XIXe siècle, le roman policier attire l'attention des lecteurs grâce à sa structure spécifique et à son raisonnement logique. Nous pouvons signaler que le roman policier contemporain a marqué une évolution observée par rapport au roman policier traditionnel dans le domaine de la caractérisation des personnages: l'enquêteur dans le roman policier traditionnel est un professionnel et un être d'exception mais dans le genre policier récent, le détective devient un personnage ordinaire plus proche du lecteur qui peut recourir à l'enquête à son gré et sans aucune obligation professionnelle. Dans le roman policier contemporain, nous pouvons distinguer trois sous genres et chaque sous-genre représente un jeu avec le lecteur.

D'une manière générale, le roman policier se base selon des codes et des règles. La codification est très importante pour éveiller le suspens qui tiendra le destinataire. Laurence Decreau souligne que: "L'ensemble vise à un effet clairement défini: piquer la curiosité du lecteur et développer sa sagacité en lui soumettant un problème dont la clé n'est pas donnée qu'à la dernière page mais qu'il est en mesure de trouver lui- même grâce à quelques indices judicieusement délivrés". Van Dine et Simenon Chandler ont déjà tracé le chemin pour les futurs auteurs du roman policier aspirant à la bonne écriture d'un roman qui satisfait l'attente et la suspense du lecteur. Bref, le roman policier est essentiellement basé sur l'observation et le raisonnement logique et le lecteur admire beaucoup ce genre littéraire parce qu'il cherche la plupart du temps à la résolution de l'énigme et il reste toujours dans un exercice de réflexion et de déduction.

\section{2-Catégories du roman policier}

Avant d'analyser la structure du roman étudié, il est nécessaire de distinguer la structure des sous genres du roman policier pour préciser ensuite le genre du roman étudié et à quel ampleur l'auteur a déjà respecté les critères structurels et narratifs du genre. 


\section{A- Le roman à énigme}

La structure du roman à énigme consiste en deux éléments essentiels: le crime et l'enquête puisque dans ce type on passe de l'énigme à la solution à travers une enquête ${ }^{5}$ ou à vrai dire dans ce type le cheminement romanesque va du mystère à l'élucidation du mystère, d'un crime à la découverte du nom du coupable à l'aide d'une enquête menée par un détective qui fait preuve de qualités d'observation et d'analyse logique ${ }^{6}$. Ce type se distingue par ce qu'on appelle le jeu intellectuel dans lequel le lecteur est invité à décrypter l'énigme, ou plutôt à découvrir l'identité du coupable avant ou en même temps que l'enquêteur ou le détective. Ainsi, la structure du roman à énigme est basée essentiellement sur deux histoires dont la première est celle du crime, alors que la seconde est celle de l'enquête. M. Lits indique que le récit d'énigme à deux notions de base: voir et dire. Le criminel a tué la victime sans être vu et ne veut pas le dire et le détective n'a pas vu mais va reconstituer l'ordre des choses par sa parole et ses investigations. Lorsque le dire va correspondre avec le voir, l'énigme sera résolue ${ }^{7}$.

\section{$\underline{\text { B- Le roman noir ou le polar }}$}

Muriel Rosemberg souligne que le polar n'est qu'un «terme par lequel on désigne le roman noir, récit réaliste du crime et du criminel par opposition au récit d'énigme ou de détection à la façon de Canon Doyle, fondé sur les prouesses intellectuelles $d u$ détective ${ }^{8} »$. Le roman noir traite des thèmes comme l'espionnage, le gangstérisme, toute puissance de l'argent, la violence, les corruptions politiques, judiciaires et policières, etc. Donc ce roman représente, selon Marc Lits, un roman policier qui «touche aux questions de sociétê ${ }^{9} »$. Le roman noir possède une structure souple où l'importance donnée au meurtrier dont le but est de savoir pour quelle raison il a déjà commis le crime. Les personnages du roman noir peuvent risquer toujours leur vie puisque l'affrontement physique reste toujours présent. Les personnages du roman noir sont souvent en conflit permanant entre leurs propres valeurs et le monde dans lequel ils vivent. Le détective est souvent privé, un homme classique qui peut se déplacer beaucoup et qui met toujours sa vie en danger. Il est solitaire et ne cherche jamais le pouvoir social et il est en conflit avec les institutions qu'il conteste. L'enquêteur se sert par des moyens plus physiques que 
La tension narrative dans le roman policier contemporain à travers Sept ans après... de Guillaume Musso

rationnels. Sa relation avec le criminel reste toujours ambiguë parce qu'il est issu du même milieu social et il utilise les mêmes pratiques ${ }^{10}$.

\section{C- Le roman à suspense}

Le roman à suspense joue essentiellement sur la révélation des sensations de la victime. Raison pour laquelle, certains théoriciens, tels que Jacques Baudou et Jean-Jacques Schleret, l'appellent «le roman de la victime» ${ }^{11}$. Sa structure est basée sur trois principes qui s'articulent, selon Reuter, comme suite :

- Un danger vital menace un personnage sympathique ;

- l'échéance est rapprochée et très vite connue ;

- le lecteur en sait plus que chacun des personnages ${ }^{12}$.

Le roman à suspense mélange la structure du roman à énigme (il y a deux histoires) et celle du roman noir (la deuxième histoire est celle qui est racontée). Dans le roman à suspense, la tension fondamentale joue sur les sentiments du lecteur. Contrairement au roman à énigme ou la démarche intellectuelle domine, le roman à suspense s'intéresse au contact émotif ou sur la subjectivité. Le lecteur, tout au long du roman, ne cesse de se demander sur le destin des personnages et sur la fin possible pour le criminel ou la victime. Dans le roman à suspense, le rôle du savoir occupe la deuxième place après celui des émotions. Pour cela, le lecteur est impuissant mais omniscient. Cette technique maintient l'envie de terminer la lecture du livre et de connaitre la fin possible des actions. Dans ce roman, l'auteur se sert des moyens stylistiques de suspension, d'expansion du texte à travers l'ajout des pauses descriptives, de dilatation du temps avant une fin inexorable. Bref, le texte narratif du roman à suspense se caractérise par "la tension narrative qui constitue une des armatures les plus profondes $d u$ récit ${ }^{13 "}$. Contrairement au roman noir, les personnages du roman à suspense n'utilisent pas leur corps comme une arme. Le héros est toujours un homme solitaire, ce qui augmente les sentiments de la souffrance. Concernant le paratexte, le titre évoque l'univers d'angoisse, des sentiments ou des termes qui évoquent le temps, les nombres, les années... La couverture montre des personnages en proie à la peur ou dans des situations dangereuses ou inquiétantes. L'univers spatial et temporel reste souvent ouvert dans le roman à suspense. 


\section{3- La tension narrative dans Sept ans après de Musso}

Après cette présentation des types et des caractéristiques des genres du roman policier, il nous semble que Sept ans après ${ }^{14}$ de Guillaume Musso appartient au roman à suspense puisque la tension narrative se pratique dès le paratexte jusqu'à la fin du texte. Le système narratif du récit se distingue par la discontinuité narrative qui se l'on trouve dans tous les endroits du roman. Le narrateur recourt à des effets stylistiques pour éveiller le suspense et la curiosité du lecteur aspirant à mettre fin satisfaisante à ses doutes et à ses émotions tendues.

\section{3-1. le paratexte}

Le terme paratexte recouvre tous les segments de texte qui apparaissent sur la couverture (titre, nom de l'auteur, de l'éditeur, texte de présentation de la quatrième couverture, informations diverses) et à l'intérieur du texte (préface, tables des matières, titres des chapitres, notes) mais également toutes les productions qui entourent le livre et se situent à l'extérieur du livre (critiques, catalogues, entretiens) désignées plus précisément sous le terme d'épitexte ${ }^{15}$.

\section{$\underline{\text { A- Le titre }}$}

Leo Hock définit le titre comme "un ensemble de signes linguistiques qui peuvent figurer en tête du texte pour le designer, pour en indiquer le contenu et pour allécher le public visé $e^{16 "}$. Le titre sert d'abord à nommer le livre où le roman qui se singularise ainsi du reste de la production littéraire. Cette fonction dénominative, comme le dit G. Genette, est suivie d'une fonction informative et incitative comme le souligne Charles Grivel: " le titre oriente et programme le comportement de lecture, ou il prémodèle un certain type de déchiffrement, ou, mis en mémoire, pendant le temps de la lecture, il réagit à tout moment sur elle, tandis qu'elle réagit sur lui, par une sorte de décryptage mutuel ${ }^{17}$." Ce choix d'un titre peu énigmatique fait accélérer le processus de l'achat du roman puisqu"il met l'accent sur une période temporelle ponctuelle qui suscite la curiosité du destinataire vers la lecture du roman pour découvrir l'énigme narrative de ces sept ans mentionnés dans le titre. L'auteur emploie également l'adverbe après qui exprime la postériorité dans le temps. Cet adverbe lui permet aussi de se poser des questions sur le passé lointain des personnages et des actions. Les points de suspension qui 
La tension narrative dans le roman policier contemporain à travers Sept ans après... de Guillaume Musso

accompagnent le titre du roman indiquent " l'interruption d'un énoncé et correspondent à une pause dans la voix ${ }^{18 "}$. Ces points de suspension permettent de mettre en valeur ce qui est dit à la suite. D'ici nous comprenons que l'auteur emploie un titre énigmatique qui permet au lecteur d'anticiper sur le contenu du texte, mais il convoque, en même temps, toute une série de questions. Ce titre du roman suscite aussi une attente, un désir auxquels la lecture du livre peut répondre.

\section{B- L'illustration}

L'illustration apparaît presque systématiquement sur les couvertures des romans policiers. Cette illustration met en scène soit la victime, soit l'agresseur, soit encore l'enquêteur ou le détective. Dans l'œuvre étudiée de Musso, nous voyons les silhouettes des deux personnages qui courent rapidement sur un pont tout en gardant le visage inquiet. Le lecteur, en voyant la couverture du roman, ne connait pas l'identité réelle de ces personnages et la raison des pas rapides. Des questions qui occupent l'esprit du lecteur en lisant le titre et en voyant la couverture du roman:

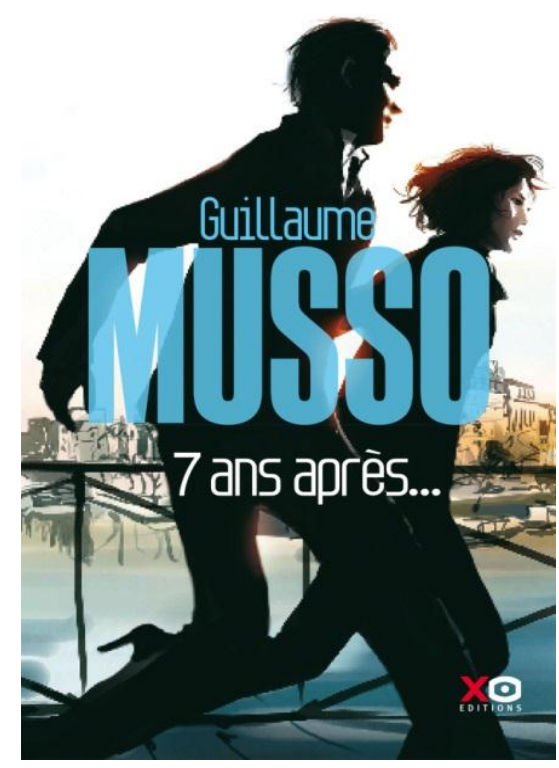


Nous remarquons ici que l'illustration renforce les effets de sens crées par le titre en mettent en valeur un des aspects qui relève de la thématique habituelle du genre. Musso par le choix d'une telle couverture du roman créait une sorte de suspense puisque le lecteur ne sait pas si ces personnages sont des coupables, des victimes ou des enquêteurs et il ne connait pas la cause de cette fuite ou de ces pas rapides et vers quoi. Effectivement, il est convaincu qu'ils sont dans une situation de danger. Cette vitesse dans la marche des personnages invite le lecteur à lire le roman pour satisfaire sa curiosité. Dans l'illustration ci-dessus nous remarquons le nom de la maison d'édition XO Editions qui se caractérise par la publication des œuvres littéraires policières et son emblème est lire pour le plaisir. Cette stratégie de la présence du nom de la maison d'édition représente une invitation explicite pour le lecteur vers la lecture d'un roman dont les actions sont pleines de suspense et de curiosité comme le dit Raphael Baroni dans $\underline{l a}$ valeur littéraire du suspense:

"Une autre façon de convertir un effet virtuel, qui ne peut être éprouvé que dans la durée de réception de l'œuvre, en une promesse pour le consommateur, est de rendre explicite l'appartenance générique de l'œuvre: dans le cas du roman policier et du roman noir, les régularités génériques sont telles que le lecteur potentiel n'a habituellement aucun doute quant à la restructuration par la curiosité ou par le suspense du récit qu'il est sur le point de consommer ${ }^{19 " .}$

De plus, nous observons que Musso emploie, dans l'illustration cidessus, trois couleurs: Le noir pour les habillements des personnages, le bleu pour le nom de l'auteur, et le blanc pour le titre du roman. Ce choix n'est jamais mis par hasard puisque le noir indique la tristesse, la mort ou le danger, ce qui signifie que les personnages du roman sont dans une situation de danger et il $\mathrm{y}$ a un mystère inconnu qu'ils s'efforcent de le déchiffrer, la couleur bleue souligne la sagesse, le rêve et la sérénité. Le bleu est une des couleurs préférées des Occidentaux. Musso veut dire, par l'écriture de son nom en bleu, que ses écrits peuvent plaire à toutes les générations et ne s'adressent pas seulement aux jeunes. Le blanc signifie la pureté, la bonté, la perfection, la vérité, l'innocence, et l'intégrité. Musso choisit la couleur blanche pour le titre du roman c'est pour dire que sept ans après la vie deviendra belle et les relations d'amour se reprendront et la paix intérieure restera l'emblème de la vie de Nikki et Sébastien, les héros du roman. Bref, le titre et l'illustration, qui fonctionnent 
La tension narrative dans le roman policier contemporain à travers Sept ans après... de Guillaume Musso

conjointement comme des clés d'accès au texte, dessinent pour le lecteur un horizon d'attente qui vient confronter le texte de

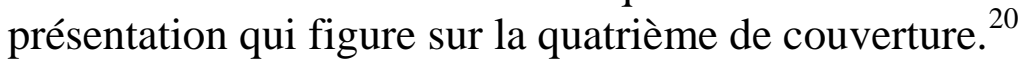

\section{C- Les écrits de quatrième de couverture}

La quatrième de couverture est souvent consultée par le lecteur averti. Sa présentation et les écrits qui y figurent varient d'un éditeur et d'une collection à l'autre ${ }^{21}$. Outre les informations déjà présentes sur la première de couverture ou sur la tranche, elle comporte des éléments qui permettent au lecteur de confirmer ou d'infirmer les hypothèses de la première de couverture. Dans la quatrième de couverture de Sept ans après, nous lisons ces phrases:

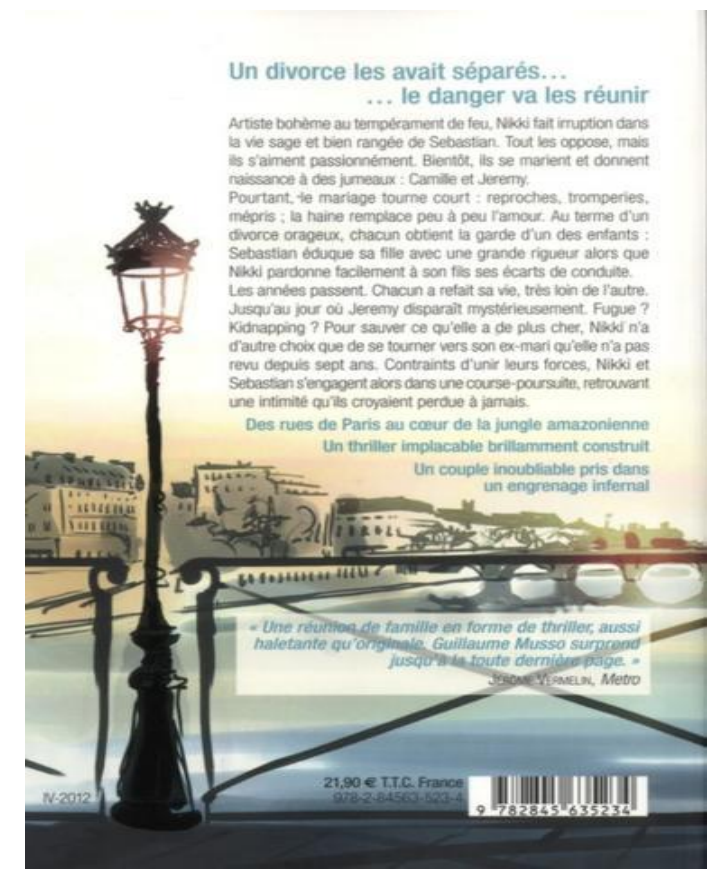

Ici l'éditeur donne un simple résumé du roman sans donner beaucoup de détails, puis il décrit le cadre spatio-temporel du roman: des rues de Paris au cœur de la jungle amazonienne, puis il mentionne le type du roman policier : un thriller implacable brillamment construit, un couple inoubliable pris dans un engrenage infernal. Ces informations encouragent le consommateur à acheter le roman puisque l'éditeur précise le type du roman en ajoutant de simples informations autour du thème principal du roman. Mais pour susciter encore le lecteur 
potentiel, l'éditeur met en bas de la quatrième de couverture, une citation positive pour le roman et ses thèmes: une réunion de famille en forme de thriller, aussi haletante qu'originale... C'est une technique qui séduit évidemment le lecteur et une garantie de la bonne qualité du roman exposé. Donc l'éditeur essaye d'inciter le consommateur à la lecture du roman par la mise en scène de la tension narrative promise dans le roman à travers la quatrième de couverture dont la finalité est double: informative pour le lecteur et commerciale pour l'éditeur et l'auteur:

"Le rôle publicitaire de la tension narrative, qu'elle s'affiche par le biais de la désignation générique de l'ouvre ou par celui de l'anticipation des incertitudes du texte dans le péritexte, paraît efficace pour attirer un grand nombre de lecteurs-consommateurs ${ }^{22}$...".

D'ici nous pouvons dire que le résumé et les citations utilisés dans la quatrième de couverture offrent les éléments d'un puzzle que seule la lecture du roman permettra d'assembler. Il s'agit de mettre le lecteur en appétit en choisissant des éléments attirants pour l'inviter à satisfaire sa convoitise. Bref, ces éléments peuvent non seulement informer le lecteur mais surtout "piquer la curiosité et suspendre le désir de lecture ${ }^{23 "}$.

\section{D- L'Incipit}

D'après Andréa Del Lungo, l'incipit est "une prise de position; un moment décisif (...) dont les enjeux sont multiples, car il doit légitimer et orienter le texte, donner des indications génériques et stylistiques, construire un univers fictionnel, fournir des informations sur l'histoire : bref, diriger la lecture. (...) L'incipit demande inéluctablement l'adhésion du lecteur à la parole du texte, ainsi qu'une implication

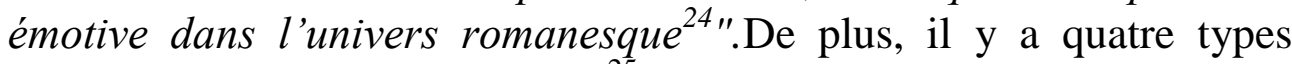
d'incipit :L'incipit informatif ${ }^{25}$, l'incipit dynamique, l'incipit progressif $^{26}$, l'incipit suspensif ${ }^{27}$.

En lisant l'incipit de Sept ans après de G.Musso, nous découvrons qu'il mélange entre l'incipit informatif, progressif et suspensif. Voici des exemples:

"Pelotonnée sous sa couette, Camille observait du fond de son lit le merle posé sur le rebord de la fenêtre. Le vent d'automne bruissait à travers la vitre, le soleil jouait entre les feuillages, (...). S'il avait plu toute la nuit, le ciel brillait à présent d'un bleu limpide qui annonçait une belle journée d'octobre ${ }^{28}$." 
La tension narrative dans le roman policier contemporain à travers Sept ans après... de Guillaume Musso

Dans ce paragraphe, le narrateur délimite le cadre temporel du roman qui se passe en octobre tout en employant des expressions métaphoriques pour décrire le vent, le soleil et la nuit. Quelques lignes plus tard, le narrateur décrit le cadre spatial du récit : "la demeure des Larbée était Située entre Madisson et Park Avenue, à hauteur de la $74^{e}$, dans une jolie traverse bordée d'arbres ${ }^{29}$." Le narrateur informe le lecteur du cadre spatio-temporel pour encadrer le récit et pour que le lecteur comprenne les événements futurs du récit. Dans le paragraphe suivant, le narrateur commence à informer le lecteur par l'introduction à l'action du récit en commençant par Sébastian, le héros du roman, qui inspecte la chambre de sa jeune fille:

"Dès qu'il fut certain que sa fille avait quitté la maison, Sébastien Larbée sortit de la salle de bain. Il pénétra dans la chambre de Camille pour son inspection hebdomadaire ${ }^{30}$."

Ici le lecteur du roman attend impatiemment ce que Sébastian va découvrir dans la chambre de Camille et il peut se demander que va-til arriver?. Cette question pousse le lecteur à accomplir la lecture du chapitre tout en attendant des événements forts du narrateur. Celui-ci saisit le moment pour suspendre encore le lecteur par l'emploi des mots qui suscitent sa curiosité et qui le laissent intéressé au futur développement du récit: "Sébastian sentit ses mains trembler. Sa colère se changea en panique au fur et à mesure que l'évidence s'imposait à lui: sa fille de quinze ans prenait la pilule 31 ". Le narrateur informe progressivement le lecteur mais en adoptant la focalisation externe pour susciter et créer chez lui un effet d'attente et de suspense. Donc l'incipit, dans Sept ans après de Musso, remplit deux fonctions $^{32}$ : fonction informative puisqu'il informe le lecteur sur l'univers romanesque tout en lui présentant les détails à peu près nécessaires sur les personnages, l'espace, ainsi que le temps. Fonction suspensive puisqu'il crée un effet énigmatique ayant pour objectif de séduire le lecteur, d'éveiller sa curiosité, de le mettre en attente de ce qui va arriver et de le pousser à lire la suite des événements. Comme tout, Il nous semble que Musso emploie dans le paratexte (titre, illustration et couverture et incipit) des éléments qui suscitent l'attente et la curiosité du lecteur vers la lecture du roman, mais si la lecture du paratexte exige un apprentissage spécifique et un lecteur cultivé, il convient de ne pas oublier que cet apprentissage n'a de sens que par rapport au texte même ${ }^{33}$. 
En un mot "si le texte sans son paratexte se ressemble à éléphant sans cornac, puissance infirme, le paratexte sans son texte est comme un cornac sans éléphant, parade inepte ${ }^{34 " .}$ Dans la suite nous présentons les stratégies que l'auteur a employées dans son texte pour accomplir sa tâche du suspense dans le roman.

\section{3-2. le texte}

\section{3-2-1. la structure régressive du récit}

Le genre policier est un genre basé sur la rétrospection. Il y a deux histoires: celle du crime et celle de l'enquête. Il en résulte une planification agencée du texte: l'histoire commence par la fin: le crime a déjà été commis lorsque commence le roman et on passe de l'énigme à la solution à travers une enquête. La deuxième histoire, celle de l'enquête qui est destinée à reconstituer la première histoire du crime. Le roman policier met l'accent sur le récit d'enquête pour pouvoir reconstituer comment un tel crime a été commis et par qui et par quels moyens. Il n'y a d'enquête et de récit d'enquête que si le récit du crime est caché. C'est l'absence du récit du crime qui appelle et permet à la fois le déroulement du récit d'enquête. Le roman à suspense respecte cette structure narrative duelle et intensifie les moments difficiles et les risques que l'enquêteur et la victime affrontent tout au long de l'histoire pour rendre le lecteur plus sympathique avec la victime et plus curieux avec l'enquêteur qui déchiffre ce puzzle mystérieux. Musso dans Sept ans après informe le lecteur dans les premiers chapitres du roman des problèmes de Sébastian avec sa fille Camille et il ne mentionne pas le crime de l'enlèvement de son fils que dans le quatrième chapitre après un appel téléphonique de Nikki, son exfemme. Musso dissimule les événements principaux du récit pour que le lecteur comprenne et compare le comportement des parents vis-àvis leurs fils. Mais il trompe le lecteur croyant que ses querelles avec sa fille d'avoir pris des pilules était le crime que l'on doit découvrir. Mais l'action principale du récit réside à l'enlèvement de Jeremy et les voyages aventureux des parents pour reprendre le fils. 
La tension narrative dans le roman policier contemporain à travers Sept ans après... de Guillaume Musso

\subsubsection{Les perspectives narratives}

\section{A- Le narrateur}

La narration renvoie aux stratégies choisies qui peuvent agencer la mise en scène de la fiction et son mode de présentation. Le type du narrateur et la perspective représentent les éléments essentiels employés pour éveiller la curiosité et le suspense chez le lecteur du roman. Le narrateur représente l'être inventé par l'auteur pour raconter l'histoire. Bien qu'il soit absent du texte, il accomplit sa tâche de mise en intrigue qui consiste à arranger et à sélectionner stratégiquement les événements ${ }^{35}$. G.Genette distingue deux types de récits: " l'un à narrateur absent de l'histoire qu'il raconte, l'autre à narrateur présent comme personnage dans l'histoire qu'il raconte. Je nomme le premier type, pour des raisons évidentes, hétérodiégétique, et le second homodiégétique ${ }^{36} \gg$. Dès lors, le narrateur peut adopter deux figures fondamentales à l'intérieur du récit qu'il raconte : soit il est "hétérodiégétique», lorsqu'il est témoin et il ne joue aucun rôle dans l'histoire racontée, soit il est «homodiégétique», quand il représente l'un des personnages de la fiction. Le narrateur, dans Sept ans après de G.Musso adopte la plupart du temps le statut hétérodiégétique:

- "Sébastian s'était replié sur lui-même, se créant un monde à sa mesure. Désormais, il quittait rarement son quartier et encore Mahhattan $^{37}$."

- "Sébastian ne venait ici que rarement. Il lui arrivait parfois d'y déposer Camille le samedi, mais il n'avait jamais mis les pieds dans l'appartement de son ex-femme.(...),il était frappé par la rapidité des changements qui effectuait le quartier ${ }^{38}$."

En lisant ces deux exemples, nous remarquons que le narrateur y indique, tout d'abord, le nom du protagoniste Sébastian, puis, il recourt à l'usage de la troisième personne du singulier «il» qui fait référence à ce protagoniste et qui a une fonction d'identification. De plus, nous observons que le narrateur reste absent des événements racontés. Il s'agit ici d'un narrateur hétérodiégétique qui ne joue aucun rôle dans les diégèses narrées. Donc le narrateur dans Sept ans après de Guillaume Musso est omniscient puisqu'il maîtrise tout le savoir et il sait plus de détails sur les héros du roman que les autres personnages. Il connait les comportements et les émotions des personnages et il peut gérer le temps et localiser l'espace. Le narrateur dans le roman étudié assume deux fonction de base : la fonction narrative puisqu'il raconte 
une histoire et présente une histoire de curiosité, et la fonction de régie ou de contrôle car il peut aisément "organiser le récit dans lequel il insère et alterne narration, descriptions et paroles des personnages ${ }^{39 "}$. De plus le changement du discours que le narrateur fait de temps en temps n'est qu'un autre argument affirmant sa fonction de régie. Pour nous transmettre les paroles des personnages, le narrateur recourt à certaines possibilités du discours tels que le discours direct, le discours direct libre, le discours indirect libre et le discours narrativisé. Voici des exemples:

\section{- Discours Direct}

Il s'agit, ici, d'une rupture dans le texte narratif puisque ce n'est plus le narrateur qui raconte, mais plutôt l'un de ses personnages. Faire parler les personnages n'est, alors, qu'un procédé qu'utilise le narrateur afin de créer du monde fictif un monde réel plein de vivacité où les personnages expriment directement leurs émotions et leurs sentiments et par conséquent le lecteur devient pris pour un témoin de leurs propos. Bref, le discours direct n'est qu'une "pause dans le discours $d u$ narrateur qui, sans cela, paraîtrait monotone ${ }^{40 "}$ :

- "Que vous est-il arrivé, monsieur Larbée?

- J'ai eu ... un accident

Inquiète ,elle décrocha son combiné

- Je téléphone à un médecin

- Ce n'est pas la peine

- Vraiment?

- Je vais bien, je vous assure, ajouta-t-il plus fermement.

- Comme vous voudrez. Je vais vous trouver des compresses et de l'alcool. Si vous désirez autre chose, faites-le moi savoir.

- Je vous remercie ${ }^{41 " .}$.

\section{- Discours direct libre}

Le discours direct libre est l'un des modes auquel le narrateur recourt pour rapporter littéralement le discours d'autrui. C'est une forme de discours direct, mais il se distingue par la suppression des marques de ponctuation ainsi que le verbe introducteur de façon que la citation se présente comme une citation directe sans aucune modification:

- "Santos prit le temps de rechercher d'autres informations pour compléter ce qu'il venait d'apprendre dans l'article. Que faire à

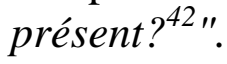


La tension narrative dans le roman policier contemporain à travers Sept ans après... de Guillaume Musso

\section{- Discours indirect libre}

Le discours indirect libre forme "une stratégie intermédiaire qui emprunte certaines de ses caractéristiques, pour une part au discours indirect, et pour une autre part, au discours direct qui conservent la qualité pragmatique de l'énoncé de départ, son expressivité ${ }^{43 "}$ :

- "Vers quels dangers étaient-ils en train de voler? Quel ennemi combattaient-ils? Pour quelle raison en voulait-on autant à Jeremy? Pourquoi avaient-ils commis la folie de ne pas demander l'aide de la police? Comment cette histoire pourrait-elle se terminer autrement qu'en prison $^{44}$

\section{- Discours narrativisé}

Dans le discours narrativisé, c'est le personnage qui disparaît et laisse raconter le narrateur, comme c'est le cas dans la citation suivante:

- "En arrivant au travail, le matin de ce 25 juillet, son supérieur hiérarchique, le commandant sorbier, lui avait annoncé sa promotion au grade de capitaine de police dans la prestigieuse Brigade nationale de recherche des fugitifs ${ }^{45}$."

- "Devant eux, un couple de Boston leurs deux enfants échangeaient des plaisanteries sur un ton complice. Derrière, un couple de japonais se chuchotait des mots d'amour à l'oreille $e^{46 " .}$.

Une telle technique nous montre que le discours narrativisé pourrait être pris pour «le degré zéro du discours rapporté ${ }^{47}$ » qui permet au narrateur d'évoquer qu'il existe un acte de parole dit par tel ou tel personnage et de lui récapituler globalement le contenu de cette parole.

\section{B- La focalisation}

Si l'étude du narrateur vise à trouver une réponse à la question :Qui raconte?, celle de la focalisation tend ainsi à répondre à la question: Qui perçoit?. Musso, dans Sept ans après, recourt à la pluralité des points de vue c'est-à-dire le récit ne se focalise pas sur le point de vue d'un seul personnage mais passe de l'un à l'autre tout au long du récit. Le lecteur suit l'intrigue à travers les héros du roman, Sébastian et Nikki mais aussi à travers les personnages secondaires comme Santos, Constance et Camille. Il y a trois types de focalisation dans le roman étudié:

\section{- Focalisation externe:}

Dans ce type, l'histoire est racontée à travers le regard d'un narrateur extérieur qui n'y participe pas. Ce narrateur ne joue aucun rôle dans le 
déroulement des événements puisqu'il "se limite aux indications qu'un observateur extérieur pourrait donner, éliminant donc toute analyse psychologique et tout dévoilement des mobiles. La scène est vue du dehors comme par une caméra, elle ressemble à un enregistrement pur et simple $48 "$ :

"Sébastian trouva une place au croisement de Van Brunt et de Sullivan Street. A cause de la circulation, il avait mis presque trois quarts d'heures pour faire le trajet jusqu'à Brooklyn ${ }^{49}$." Ici le narrateur raconte une histoire d'où il est absent et son rôle est limité à décrire la scène de l'aller-retour de Sébastian. Le rôle du narrateur est restreint et il se ressemble à l'oculaire d'une caméra qui filme et enregistre les faits et les gestes des personnages de l'extérieur.

\section{- Focalisation zéro}

La focalisation zéro représente une absence de focalisation parce que le narrateur connait le passé, le présent et le futur de tous les personnages. Il a un regard omniscient et il peut présenter en détail les faits et la psychologie des personnages. Dans cette absence de focalisation, le lecteur possède plus d'informations que les autres personnages du roman puisque "la scène est rapporté par un auteur omniscient, qui voit tout et ne se trompe jamais ${ }^{50 ":}$

"Dans les années 1990, Nikki avait été arrêtée à plusieurs reprises pour différents vols, ivresse sur la voie publique et possession de stupéfiants. Si elle n'avait jamais été incarcérée, elle avait payé de multiples amendes et effectue plusieurs dizaines d'heures de travaux d'intérêt général. Sa dernière infraction remontait à 1999. Depuis, elle s'était tenue tranquille ${ }^{51 " .}$

"La nécessité de fixer des principes pour élever leurs enfants avait au contraire exacerbé les conflits. Nikki concevait l'éducation sur un mode privilégiant la liberté et l'autonomie. Sébastian ne l'avait pas suivie sur ce chemin qu'il jugeait dangereux. Il avait cherché à la convaincre que seules des règles strictes structuraient la personnalité d'un enfant ${ }^{52 "}$.

Dans les deux exemples ci-dessus, le narrateur raconte le passé de Nikki et les amendes qu'elle a payées. Le narrateur cite la date exacte des crimes et les amendes, ce qui signifie qu'il maîtrise une connaissance très forte sur le passé et le présent et le futur des personnages. Dans le deuxième exemple, le narrateur recense les causes du divorce entre Nikki et Sébastian. Il affirme que le système éducatif et familial joue un bon rôle à cette divergence de vue puisque 
La tension narrative dans le roman policier contemporain à travers Sept ans après... de Guillaume Musso

Sébastian est "le produit d'une éducation élitiste et bourgeoise ${ }^{53 "}$ mais Nikki a un gout mondain et elle "vivait au jour le jour, dans l'insouciance et la désinvolture ${ }^{54 " . ~ P a r ~ r e c o u r s ~ a ̀ ~ l a ~ f o c a l i s a t i o n ~ z e ́ r o, ~ l e ~}$ narrateur se distingue alors par l'omniscience et l'omniprésence. Il connait les moindres détails plus que les personnages eux-mêmes; il connaît leurs comportements, leurs pensées, leurs émotions, leurs secrets, voire leurs rêves. Il peut aussi raconter leur présent, leur passé, ainsi que leur futur. Bref, le narrateur, en adoptant son propre point de vue, " ne pratique aucune restriction de champ et n'a donc pas à sélectionner l'information qu'il délivre au lecteur ${ }^{55 " .}$

\section{- Focalisation interne}

La focalisation s'effectue selon le point de vue d'un acteur intérieur dans la diégèse racontée. Dans cette perspective, le narrateur est sur le même niveau de l'information que le «personnage focal ${ }^{56} »$ En d'autres termes, le narrateur ne peut raconter les événements que dans le cadre de l'optique du focalisateur et il ne peut pas le dépasser:

"Constance sentit que les larmes montaient. Elle aimait tellement ce jardin avec son figuier, son abricotier, sa haie de lilas...Dès les premières secondes de sa visite avec l'agent immobilier,(...), elle avait su que c'était ici qu'elle voulait vivre... et peut être un jour y élever un enfant(...). Dévasté par l'injustice de la situation, elle éclata en sanglot. Elle avait beau de dire que la mort était inéluctable(...). La situation lui semblait surréaliste ${ }^{57 " .}$

Dans l'exemple ci-dessus, le narrateur adopte la vision de Constance sur la vie. Elle a déjà rêvé d'avoir une belle maison avec un jardin riche mais sa maladie cancéreuse arrête ses ambitions et la rend désespérée et découragée et elle n'admet jamais l' idée de la mort. Le narrateur ne peut pas dépasser cette vision du personnage et n'ajoute pas au lecteur d'autres détails supplémentaires. Le verbe "lui semblait" à la fin de cet extrait affirme le respect des frontières narratives de la part narrateur pour ses narrataires.

\section{3-2. 3. Vitesse du récit}

L'étude de la vitesse est, en effet, destinée au traitement du rapport établi entre la durée fictive des événements en années, mois, jours, heures et la durée de la narration ou plus exactement de la mise en texte, exprimée en nombre de pages ou de lignes ${ }^{58}$. Dans Sept ans après de Musso, le rythme du récit est dominé par les quatre procédés 
traditionnels (ellipse, sommaire, pause, et scène) que cite Genette dans son ouvrage intitulé Figures III et dont les deux premiers visent à accélérer la narration, alors que les deux derniers ont pour but de la ralentir.

\section{A. L'Ellipse}

L'ellipse n'est qu'une technique autorisant au narrateur de faire une accélération maximale du récit ${ }^{59}$. Genette souligne que "l'analyse des ellipses se ramène à la mise en considération du temps d'histoire élidé, et la première question est ici de savoir si cette durée est indiquée (ellipses déterminées) ou non (ellipses indéterminées) ${ }^{60 "}$.

En lisant le roman de Musso, nous apercevons l'adoption de deux types d'ellipses: déterminées et d'ellipses indéterminées.

\section{- Des ellipses déterminées:}

- "deux ans plus tard, tout avait commencé dans le sang, tout finirait dans le sang ${ }^{61 " .}$

- "Trois mois auparavant, le jour de ses trente-sept ans, Constance avait appris trois nouvelles: deux bonnes et une mauvaise ${ }^{62 " .}$

Dans ces deux exemples, il s'agit des omissions comptées à partir desquelles le lecteur peut préciser le laps omis que le narrateur passe sous silence. Le lecteur ne sait pas ce que les protagonistes du roman ont réalisé pendant les deux ans omis par le narrateur dans le premier exemple et il est informé de quelques nouvelles sur la vie de Constance dans le deuxième exemple. Le narrateur accélère le rythme du récit en vue de capter l'attention du lecteur sur des événements précis et orientés selon son propre choix.

\section{- Des ellipses indéterminées}

les ellipses indéterminées s'attachent aux indications temporelles qui donnent au lecteur l'impression qu'il existe une omission dans le temps de l'histoire racontée, mais sans pouvoir préciser en définitive la durée élidée:

"Au fil des années, Sébastian avait remis de l'ordre dans sa vie. Désormais, Nikki n'était plus qu'un lointain souvenir ${ }^{63 " . ~ L e ~ n a r r a t e u r ~}$ ne mentionne pas le nombre exact de ces années mais il donne presque un résumé de la vie de Sébastian après son divorce.

Il y a un autre type d'ellipse que le lecteur peut observer par le contexte ou bien par l'enchaînement des événements dans le roman. Ce type s'intitule l'ellipse implicite dont la présence "n'est pas déclarée dans le texte et que le lecteur peut seulement inférer de quelque lacune 
La tension narrative dans le roman policier contemporain à travers Sept ans après... de Guillaume Musso

chronologique $^{64}{ }$. Nous pouvons remarquer cette ellipse entre la fin d'un chapitre et le début d'un autre:

Quand Constance Legrand poursuivait Sébastian et Nikki pour les arrêter:

"Elle fit un ultime effort pour s'accrocher à son pistolet, mais le monde autour d'elle vacilla. Puis tout devint noir et elle s'évanouit ${ }^{65 " .}$

Mais dans le debut du chapitre suivant nous pouvons lire:

"Lorsque Constance reprit connaissance, elle eut la surprise de se retrouver...dans son propre lit $^{66 " .}$.

Le lecteur remarque que Constance Legrand, dans le chapitre précédent, était dans la rue et elle s'évanouissait mais dans le chapitre suivant elle était dans son propre lit, ce qui pousse le lecteur à se poser la question: qui l'avait ramenée chez elle? Les pompiers? Le SAMU? Ou Botsaris? L'absence d'une réponse directe au sein du texte invite le lecteur à suivre la lecture des autres chapitres pour essayer de trouver des indices permettant de combler ce manque d'informations. Ce changement spatial a pour objectif de nous montrer l'élision d'un certain laps inexprimé que Constance a passé afin de se déplacer de la rue à sa maison. Bref, les ellipses marquent le degré maximal de l'accélération du temps puisque des minutes, des heures, des jours, des semaines, des mois, ainsi que des années peuvent être condensés dans une absence totale de la narration.

\section{B- Le sommaire}

Le narrateur tend à utiliser cette technique en vue de récapituler en quelques mots de plusieurs minutes, heures, journées, mois ou années. J. Vincent souligne que «le sommaire résume une longue durée d'histoire en quelques mots ou quelques pages ${ }^{67} »$. Si le narrateur recourt à l'ellipse en vue de produire, dans le récit, une accélération maximale, c'est par recours au sommaire qu'il procède à ralentir un peu cette accélération. Nous pouvons lire ces deux exemples:

- "Pendant des heures, elle avait épluché tous les documents en sa possession, en quête d'un indice qui l'aiderait à remonter la piste du couple d'Américains ${ }^{68 "}$.

- "Il fallut plus d'une heure à Nikki et Sébastian pour détailler à Constance l'enchaînement des événements qui avaient bouleversé leur vie ces derniers jours ${ }^{69}$.". 
Nous observons que le narrateur présente un exposé sommaire par lequel le narrateur condense, en quelques phrases, des événements qui pourraient occuper une durée assez longue. Le lecteur remarque que ces propos sont racontés dans un style narrativisé, ce qui permet au narrateur d'ajouter des commentaires ou des explications pour renforcer l'effet du réel ${ }^{70}$. Les deux passages ci-dessus nous révèlent comment le narrateur a pu réduire, dans le temps du récit, le contenu des dialogues dont la durée remplit, certes, des heures dans le temps de l'histoire.

\section{La scène}

A l'opposition de l'ellipse et du sommaire dont la fonction est d'accélérer le rythme du récit, la scène est l'un des procédés dont se sert le narrateur afin de ralentir ce rythme. Produire une sorte d'équivalence, ou plutôt d'égalité entre le temps raconté et le temps mis à raconter est, en effet, la fonction essentielle de cette technique. Les dialogues sont, à ce propos, les meilleurs exemples affirmant la présence de la scène dans le roman de Musso comme le signale Yves Reuter : "Les dialogues instaurent une impression d'équivalence entre rythme de la fiction et rythme de la narration ${ }^{71}{ }^{»}$. Prenons ce dialogue comme un exemple de la scène:

-" Attends, tu ne sais pas tout, Sébastian

- Quoi encore?

- Jeremy a déjà eu un petit problème: il a un casier judiciaire.

Il resta sans voix et la regarda avec incrédulité.

- Tu plaisante? Et tu ne m'as jamais mis au courant?

- Il a fait des conneries ces derniers jours?

- Quel genre de conneries?

- Il y a six mois, il s'est fait serrer par une patrouille en train de taguer un camion de livraison dans le hangar d'Ikea ${ }^{72 " .}$

Alors que ce dialogue tenu entre Sébastian et Nikki ne remplit que quelques instants dans la fiction romanesque, il occupe deux pages dans le roman. Il convient, donc, de mentionner que c'est par recours à la scène que le narrateur ne cherche qu'à ralentir la vitesse du récit.

\section{La pause}

Dans la pause, le narrateur "va jusqu'à arrêter le temps événementiel pour décrire un personnage ou un paysage ${ }^{73 "}$. Par cette technique narrative, le narrateur peut incorporer des passages descriptifs ou 
La tension narrative dans le roman policier contemporain à travers Sept ans après... de Guillaume Musso

ajouter des commentaires personnels très longs. Nous pouvons lire nombreux passages où le narrateur se sert de pause narrative:

"Des parfums de cuisine portoricaine flottaient dans l'air graisseux. Des rythmes caribéens s'échappaient des fenêtres. Des drapeaux dominicains ornaient le perron des maisons. Nul ne pouvait ignorer bien longtemps que Brunswick était un fief latino. Tentaculaire, le quartier s'étendait sur des dizaines de blocs et avait gardé un coté rugueux(...). Ici, pas de jeunes friqués, d'artistes à la mode ou de restaurants bio, mais une succession d'entrepôts, de maisons aux toits de tôle, d'immeubles en brique, de murs couverts de graffitis et de terrains vagues envahis de mauvaises herbes ${ }^{74}$."

Le narrateur décrit le bar que Jeremy fréquentait avant son absence. Sébastian fait tout le possible pour rencontrer le directeur du bar afin de retrouver son fils enlevé. Le narrateur décrit une banlieue sinistre et dangereuse. Il nous semble que le récit ne progresse pas et le narrateur ajoute des informations pour le lecteur sur l'espace terrible:

"Le bar était repeint à l'hémoglobine. Le parquet maculé de taches noires et collantes. Les murs de brique aspergés de mélasse pourpre. Les boiseries souillées. Les éclaboussures avaient atteint jusqu'aux étagères qui débordaient de bouteille derrière le comptoir ${ }^{75 " . ~}$

Cette description ne fait pas avancer les événements dans le roman mais elle peut augmenter le sentiment de la peur et de panique chez le lecteur souhaitant lire les futures lignes du roman pour connaitre la fin d'un tel chapitre. Nous pouvons distinguer entre la scène et la pause. Cette distinction réside dans le fait que le narrateur se sert de la scène pour décrire minutieusement tel ou tel acte faisant partie de l'histoire racontée tandis qu'il consacre la pause à des descriptions qui n'ajoutent rien sur le plan historique, mais qui visent à faire progresser le plan textuel. Mais à travers ces deux techniques, le narrateur crée un ralentissement dans le récit. Ces quatre procédés narratifs permettent au lecteur de comparer entre le temps du discours et le temps de l'histoire. Musso les a utilisés dans Sept ans après pour éveiller chez le lecteur un suspense narratif représenté dans l'accélération ou dans le ralentissement des événements racontés.

\section{4- Des éléments cinématographiques}

Musso a recours dans son roman à des procédés cinématographiques pour accrocher le lecteur, lui donner envie de compléter la lecture et 
entretenir la tension narrative. Il n'est guère étonnant que l'auteur se serve de techniques cinématographiques, car son roman est ancré dans la culture du cinéma: Jeremy rêve d'être réalisateur et Musso fait allusion à un nombre considérable de films comme: le Fabuleux Destin d'Amélie Poulain (2011) de Jean Pierre Jeunet et le Da Vinci Code (2006) de Ron Howard (cinéaste américain). Ces techniques cinématographiques se représentent dans l'emploi du cliffhanger et le flash-back dans le texte narratif du roman:

\section{4-1. Le cliffhanger}

Le cliffhanger est une stratégie narrative consistant à introduire une rupture dans le récit au moment où le suspense est à son comble. $\mathrm{Ce}$ processus consiste à désynchroniser la fin textuelle de la résolution de l'intrigue. Ce cliffhanger peut se baser sur la création d'un effet de suspense ou de curiosité et il est souvent suivi d'une explicitation des doutes laissés en suspens et de commentaires métadiégétiques invitant le lecteur à attendre la suite du récit. Nous remarquons des cliffhangers dans certains récits folkloriques, comme les récits suspendus de Shéhérazade dans Les $1001^{76}$. Cette technique narrative peut être considérée comme une technique commerciale qui pousse le lecteur à accomplir le roman ou le film jusqu'à la fin. Dans Sept ans après de Guillaume Musso, nous remarquons l'emploi intense de ce cliffhanger qui encourage le lecteur à ne pas laisser le roman qu'en décryptant l'énigme. Ce cliffhanger se pratique toujours à la fin du chapitre:

"Une série de numéros indiquait l'ordre de prise de chaque cachet.(...). Sa colère se changea en panique au fur et à mesure que l'évidence s'imposait a lui: sa fille de quinze ans prenait la pilule ${ }^{77 " .}$.

Cette tension narrative qui se pratique dès le premier chapitre ne peut pas être résolue que plus tard. Le narrateur oblige le lecteur à suivre le chapitre suivant du roman pour découvrir l'énigme présente au chapitre précédent mais ce narrateur hétérodiégétique ajoute un deuxième cliffhanger qui ne fait qu' alourdir le nombre des puzzles dans le roman:

"Reprenant ses esprits, elle attrapa son sac, bien décidée à ne pas rester une seconde de plus en présence de son père. Sébastian essaya de la retenir, mais elle le repoussa et sortit de la maison sans même refermer la porte derrière elle ${ }^{78 "}$. 

après... de Guillaume Musso

Le lecteur se demande alors ou va-t-elle? Chez sa mère? chez son copain? chez sa grand- mère? Cette série de questions complique la situation narrative pour lecteur et l'accable d'une bonne doses de mystères. Ce lecteur ne peut jamais laisser la lecture du chapitre qu'en lisant le chapitre suivant pour satisfaire sa curiosité et faire arrêter ce cycle vicieux du suspense. Ce principe peut s'appliquer de même au cinquième chapitre où Sébastian découvre le cadavre mutilé de Decker dans le bar, et il se retrouve face à un guerrier maori. Le chapitre s'achève sur ces mots qui invitent le lecteur à tourner vite la page pour connaitre le sort et le danger qui menacent le protagoniste:

" Tetanisé, Sébastian resta cloué sur place. Il ne leva même pas les bras pour se protéger lorsque la lame s'abattit sur lui ${ }^{79} . "$

Cette stratégie de l'emploi du cliffhanger stimule à éveiller et maintenir vivace l'intérêt du destinataire. Celui-ci est encouragé à attendre impatiemment le chapitre suivant pour satisfaire ses attentes concernant les événements. Les hypothèses du récepteur se caractérisent alors par une anticipation teintée de doute et d'incertitudes et il souffre de ce sentiment de méfiance et d'inquiétude jusqu'à la fin du roman. Nous pouvons remarquer que le narrateur adopte la focalisation externe dans le cliffhanger pour limiter les informations données au lecteur et pour susciter chez lui la curiosité et le suspense. Pour cela la focalisation externe est toujours adoptée dans cette stratégie narrative ${ }^{80}$ Bref, cette technique se crée par le contraste entre une narration tonique et une narration atone 81 c'est-à-dire entre un narrateur qui alimente le texte par des éléments dramatiques et énigmatiques et un lecteur impatient qui espère trouver la réponse immédiate à ses questions implicites ou explicites qui deviennent insupportables par la notion du retard temporel imposé par le texte:

"Pour garder l'intérêt du lecteur jusqu'au final, il faut que la distribution de l'information soit dosée judicieusement aux moments idoines, et la réponse principale doit être dissimulée jusqu'au dénouement ${ }^{82 "}$.

\section{4-2- Le flash-back}

le flash-back, appelé aussi analepse ou anaphore, permet au narrateur de faire des retours en arrière explicatifs ${ }^{83}$. Il peut interrompre le déroulement d'un récit par le rappel des événements passés. Il aide à raconter un souvenir, un événement. Ce procédé, qui appartient à la 
cinématographie, s'est étendue à la littérature depuis plusieurs années. Les flashs-back sont utilisés dans le roman afin de faire comprendre des détails des chapitres précédents embrouillés. G. Musso pratique ce flash-back tout au long du roman soit pour atténuer l'acte dramatique, soit pour ralentir la vitesse narrative du récit, soit pour augmenter le suspense chez le lecteur. Nous pouvons distinguer deux types d' analepses: celles externes et celles internes.

\section{- Le flash-back externe}

Dans Sept ans après de Musso, le flash-back externe est plus fréquent que le flash-back interne. Le narrateur s'attache toujours à évoquer dans le roman des moments rétrospectifs qui précèdent le temps central indiqué au début de l'action. Ces analepses externes sont effectivement liées à deux fonctions essentielles. D'une part, le narrateur s'en sert en vue de mettre en relief la vie professionnelle et familiale de quelques personnages et les aventures vécues.

Le narrateur consacre deux chapitres complets pour nous retracer la vie de Sébastian et Nikki tout en nous informant sur leur éducation et leur origine sociale puis il nous transmet l'histoire de leur connaissance et de leur amour mutuel tout en précisant les lieux et les temps de leur rencontre:

"De Broadway à la $7^{e}$ Avenue, Macy's occupe tout un pâté de maison. En ce 24 décembre, le plus grand magasin du monde est bondé. La neige qui tombe dru depuis le début de l'après-midi n'a dissuadé ni les New-Yorkais ni les touristes de venir faire leurs derniers achats avant le réveillon ${ }^{84}$." Le narrateur délimite d'abord le cadre spatio-temporel de l'action pour informer le lecteur qu'il est devant une scène et une histoire différente. Les chapitres qui précèdent cette technique du flash back, qui est centré sur Sébastian et Nikki, sont pleins du cliffhanger narratif puisque Nikki reçoit un courriel qui contient une vidéo concernant leur fils enlevé. Le narrateur fait allonger le texte par des histoires qui concernent des personnages secondaires comme Santos et Drake Decker. Le lecteur devient tout à fait impatient et il est choqué par une narration qui recense les aventures des protagonistes à Paris cherchant l'origine de cette vidéo dans l'espoir de négocier l'expéditeur pour retrouver leur fils perdu. Puis le narrateur fait augmenter cette tension narrative par un autre flash-back destiné à rappeler la vie de Nikki il y a dix-sept ans plutôt et comment les deux protagonistes ont célébré leur mariage ${ }^{85}$. Nous pouvons trouver d'autres analepses dans 
La tension narrative dans le roman policier contemporain à travers Sept ans après... de Guillaume Musso

les pages postérieures du roman. Elles sont présentes dans les conversations enregistrées par Dr Marion Crane avec Jeremy. Santos les découvre par hasard à la suite d'une recherche sur le téléphone de Jeremy. Celui-ci a été accusé d'avoir volé de jeux vidéos et le juge l'a obligé d'un suivi psychologique ${ }^{86}$. Alors, le narrateur se sert de cette analepse en vue de rappeler la vie antérieure des personnages et pour que le lecteur comprenne le passé lointain des criminels, des détectives, et des protagonistes.

\section{- Le flash back interne}

Le narrateur recourt au flash-back interne pour "combler après coup une lacune antérieure du récit, lequel s'organise ainsi par omission provisoire et réparation plus ou moins tardives ${ }^{87 " . ~ G . ~ G e n e t t e ~ l e s ~}$ nomme des analepses complétives ou renvois ${ }^{88}$. Le narrateur emploie de temps en temps ce type d'analepse dans le roman de Musso:

"Alors que le taxi continuait le long des quais rouillés, Sébastian se frotta les paupières, essayant de reconstituer l'enchainement des événements. Après avoir tué Memphis, les hommes du cartel avaient envoyé l'un de leurs(...) pour prendre contact avec Drake Decker. Sous la menace, Drake avait dû avouer s'être fait voler le baladeur par un gamin appelé Jeremy.(...). Et c'est par l'intermédiaire de la page Facebook du groupe de rock que Flavia avait réussi à remonter la piste jusqu'à Jeremy(...)." 89

Cette rétrospection est donc interne puisque tous les événements évoqués se passent au cours du champ temporel du roman et ne précèdent pas le point central indiqué dans l'incipit. Par conséquent, le lecteur comprend l'histoire de Jeremy et sa relation avec le cartel brésilien. Ce flash-back a une fonction complétive; il évoque des moments élidés dans l'histoire racontée dans le but de reconstituer l'histoire de l'enlèvement de Jeremy et pour la compréhension totale du texte.

Il nous semble que le flash-back pratiqué par le narrateur peut représenter une pause narrative ou un éclaircissement pour le lecteur sur le passé des personnages. Cette anaphore peut également augmenter le suspense chez un lecteur impatient qui espère trouver une réponse rapide du cliffhanger déjà pratiqué par le narrateur. Bref, le cliffhanger, le flash-back et la mise en abyme aident à bien construire le texte romanesque et à rendre le lecteur toujours vigilant à l'égard des événements du roman. 


\section{4-3- La mise en abyme}

la mise en abyme est un procédé par lequel on intègre dans un récit, dans un tableau, un élément signifiant de ce récit ou de ce tableau, qui entretient avec l'ensemble de l'œuvre une relation de similitude. Musso dans Sept ans après insère le type d'une comédie intitulée le screwball Comedy, qui est un sous genre de la comédie hollywoodienne déjà célébrée dans les années 1930. C'est dans le but d'informer le lecteur de la thématique abordée et de l'aider à bien lire et critiquer le roman comme l'exprime franchement Musso dans son roman: "Avec Nikki, la vie prenait l'allure d'une screwball comedy: Il était Cary Grant, elle était Katherine Hepburn ${ }^{90 " .}$. Cary Grant et Katherine Hepburn sont des célèbres acteurs du cinéma américain. Musso adopte cette technique narrative dans le roman pour montrer les similitudes entre le réalité et la fiction ou à vrai dire entre des héros fictionnels et des personnages réels.

\section{5- L'espace et la tension narrative}

\section{5-1. la ville}

La ville occupe une place primordiale dans le roman policier. La plupart du temps nous observons dans Sept ans après le décor de grandes villes puisque nous assistons à un univers romanesque toujours ouvert et à une fréquence des déplacements, des poursuites, et des voyages de la part des personnages. Musso choisit l'Amérique, la France et le Brésil comme cadre spatial da sa fiction policière. Il s'intéresse à montrer les spécificités de chaque lieu comme si le lecteur est au centre de l'action. Musso ne se contente pas de mentionner les villes mais aussi il cite de noms de quartiers, de rues, et de magasins pour que le lecteur élabore le monde fictif à partir des éléments du monde réel. Musso emploie les toponymes des rues, des magasins et des lycées pour fournir au lecteur qui ne connait pas l'Amérique les informations qui lui permettent de découvrir l'espace de l'action romanesque :

"Son lycée, Le St. Jean Baptiste High School, était un établissement pour filles. Une école d'élite accueillant la jeunesse dorée newyorkaise ${ }^{91} . "$

Mais pour suspendre le lecteur, le narrateur choisit des quartiers dangereux dans les plus belles villes du monde, c'est dans le but d'accomplir la tension narrative pratiquée par le texte. Le narrateur donne des informations sur la station Barbès-Rochechouart à Paris. 
La tension narrative dans le roman policier contemporain à travers Sept ans après... de Guillaume Musso

Cet endroit est réputé par les mauvaises qualités: vol, enlèvement, chaos, et meurtre.:

"Barbès... Dès qu'il fut dans la rue, Sébastian découvrit un Paris qui n'était pas celui des cliches. Ici, pas de passants en béret trimbalant leur baguette sous le bras, pas de fromagerie ou de boulangerie traditionnelle à chaque coin de rue. Ce n'était pas non plus le Paris de la tour Eiffel ou de l'Arc de triomphe, mais un Paris multiethnique, rugueux et coloré qui lui rappela le melting-pot newyorkais. ${ }^{92}$

Ici le narrateur critique le quartier et choisit un tel lieu pour éveiller le suspense chez le lecteur même dans les villes classées les plus modernes au monde. Le narrateur souligne encore que l'Amérique et la France ne représentent pas toujours un cadre spatial rose mais le crime existe même dans les endroits les plus prestigieux du monde. Le narrateur choisit encore la ville de Rio de Janeiro au Brésil comme le troisième cadre spatial de la fiction romanesque. Cette ville est célèbre dans le monde entier pour son carnaval et ses plages pittoresques et le nombre des touristes qui la visitent régulièrement. Mais cette deuxième grande ville au Brésil est gangrenée par le taux élevé du crime et de la violence et" l'état de Rio reste l'un des endroits les plus dangereux au monde. Son taux d'homicide, trente fois supérieur à celui de la France ${ }^{93}$,..."

Le choix de Musso pour des quartiers qui sont touchés par la violence et le danger représente un facteur aidant à cette tension narrative du texte et ce choix peut avoir deux fonctions: L'une informative puisque le lecteur est invité à explorer des endroits peu visités par lui et une fonction typologique puisque le narrateur choisit des lieux classés dangereux comme scène du crime.

Le narrateur se sert d'autres moyens linguistiques, propres aux villes parcourues par les héros du roman, qui peuvent augmenter cette tension narrative. Il recourt de temps en temps à l'insertion des mots et des expressions étrangères sans aucun accompagnement de l'équivalent français et aussi à l'emploi intensif des points de suspension: 
- - Des mots anglais:

\begin{tabular}{|l|l|l|l|l|}
\hline $\begin{array}{l}\text { Sprint } \\
\text { p.15 }\end{array}$ & $\begin{array}{l}\text { Notebook } \\
\text { p.44 }\end{array}$ & $\begin{array}{l}\text { Hot dogs } \\
\text { p.53 }\end{array}$ & Book p.170 & Pin-up p.318 \\
\hline $\begin{array}{l}\text { Preppy } \\
\text { p.16 }\end{array}$ & $\begin{array}{l}\text { Shooter } \\
\text { p.44 }\end{array}$ & $\begin{array}{l}\text { Twitter, } \\
\text { Facebook } \\
\text { p.73 }\end{array}$ & Casting p.170 & Design p.331 \\
\hline $\begin{array}{l}\text { Geek } \\
\text { p.41 }\end{array}$ & Shit p.46 & Poker p.144 & $\begin{array}{l}\text { Business as } \\
\text { usual p.172 }\end{array}$ & SMS p.343 \\
\hline $\begin{array}{l}\text { Nuggets } \\
\text { p.41 }\end{array}$ & Teck p.47 & $\begin{array}{l}\text { No agression } \\
\text { Sir p.148 }\end{array}$ & Tuk-tuk p.276 & $\begin{array}{l}\text { Patchwork } \\
\text { p.384 }\end{array}$ \\
\hline $\begin{array}{l}\text { Geek } \\
\text { p.42 }\end{array}$ & $\begin{array}{l}\text { Food trucks } \\
\text { p.53 }\end{array}$ & Le cash p.155 & $\begin{array}{l}\text { Debriefing } \\
\text { p.295 }\end{array}$ & $\begin{array}{l}\text { Monitoring } \\
\text { p.386. }\end{array}$ \\
\hline
\end{tabular}

\section{•- Des mots familiers et populaires et leurs sens}

\begin{tabular}{|c|c|c|c|c|c|}
\hline$\underline{\text { Le mot }}$ & $\underline{\text { Le sens }}$ & $\underline{\text { Le mot }}$ & $\underline{\text { Le sens }}$ & $\underline{\text { Le mot }}$ & $\underline{\text { Le sens }}$ \\
\hline $\begin{array}{l}\text { Mouchard } \\
\text { p. 48: }\end{array}$ & espion & $\begin{array}{l}\text { Coincer } \\
\text { p. } 49\end{array}$ & rencontrer & $\begin{array}{l}\text { Piger } \\
\text { p.51 }\end{array}$ & comprendre \\
\hline $\begin{array}{l}\text { Roublardise } \\
\text { p. } 48\end{array}$ & malice & $\begin{array}{l}\text { Bosser } \\
\text { p.50 }\end{array}$ & travailler & $\begin{array}{l}\text { Dope } \\
\text { p.51 }\end{array}$ & drogue \\
\hline Tôle p.50 & prison & $\begin{array}{l}\text { Bellâtre } \\
\text { p.50 }\end{array}$ & homme & $\begin{array}{l}\text { Fric } \\
\text { p.55 }\end{array}$ & argent \\
\hline $\begin{array}{l}\text { Saouler } \\
\text { p.57 }\end{array}$ & ennuyer & $\begin{array}{l}\text { Frime } \\
\text { p.57 }\end{array}$ & simulation & $\begin{array}{l}\text { Nana } \\
\text { p.57 }\end{array}$ & copine \\
\hline $\begin{array}{l}\text { Choper } \\
\text { p.57 }\end{array}$ & prendre & $\begin{array}{l}\text { Daigne } \\
\text { p.57 }\end{array}$ & $\begin{array}{l}\text { allure } \\
\text { ridicule }\end{array}$ & $\begin{array}{l}\text { Guignol } \\
\text { p.57 }\end{array}$ & $\begin{array}{l}\text { individu } \\
\text { grotesque }\end{array}$ \\
\hline Gosse p.57 & enfant & $\begin{array}{l}\text { Foutaises } \\
\text { p.303 }\end{array}$ & bêtises & $\begin{array}{l}\text { Boulot } \\
\text { p.308 }\end{array}$ & travail \\
\hline
\end{tabular}

\begin{tabular}{|l|l|}
\hline •- Des mots potugais & \\
\hline Le mot ou l'expression en portugais & Le sens en français \\
\hline - Siga a seta! P.374 & Suivez la flèch! \\
\hline Vamos com cuidado! p.374 & Faisons attention! \\
\hline Levante-se! P.374 & \\
\hline Ele esta morto! p.378 & - leve-toi \\
\hline
\end{tabular}

\section{$\underline{\text { 5-2 Les points de suspension }}$}


La tension narrative dans le roman policier contemporain à travers Sept ans après... de Guillaume Musso

Nous observons l'emploi massif des points de suspension dans le roman. Ces points suspensifs remplissent quelques fonctions essentielles:

1- Une pause dans une phrase parlée ou une phrase interrompue. Dans d'autres cas, le locuteur ne finit pas son énoncé, elle s'interrompt ou se fait couper la parole par un interlocuteur:

- " je ne sais plus. J'ai contacté les services des principaux hôpitaux, J'ai...

- Tu n'as rien trouvé de bizarre en fouillant sa chambre? ${ }^{94 "}$

2- Une énumération incomplète puisque on peut nommer quelques exemples et les fait suivre par des points de suspension pour bien montrer que la liste pourrait se poursuivre

- Tout sentait mauvais dans cette histoire: des cercles de jeu illégaux impliquant des mineurs, une fugue, des dettes potentielles... ${ }^{95}$

3- Hésitation du locuteur:

" Elle sentit son cour s'accélérer tandis que de fines gouttes de transpiration perlaient sur son front.

- Je... je faisais un peu de ménage, c'est tout ${ }^{96 "}$

- "Que vous est-il arrivé, monsieur Larbee?

- J'ai eu... un accident"

4- des phrases supprimées et des mots inachevés:

- "Si quatre-vingts- dix kilos valent 5,2 million, un kilo vaut...

- ... un peu moins de 60000 dollars, compléta Joseph. Tu peux m'expliquer mainte ${ }^{98}$..."

Parfois les locuteurs dans le roman laissent le lecteur deviner et compléter leurs paroles par l'emploi des points de suspension:

"- Croyez bien que c'est un cas d'urgence et que nous prendrons bien soin de votre ${ }^{99}$...".

Le locuteur ignore le mot sécurité à la fin de la phrase pour éveiller la curiosité du lecteur et le rendre toujours vivace à l'égard des paroles des personnages. Pour cela le lecteur n'est pas considéré un simple récepteur de l'acte diégétique mais aussi un héros et un personnage qui peut combler le vide du texte et peut déchiffrer les idées implicites des personnages.

5- D'autre part, les points des suspension peuvent éviter la répétition dans le texte, c'est dans le but de ne pas toucher le lecteur d'un 
sentiment de la monotonie et le rendre toujours passionné à l'action du roman:

"Bien sûr que je m'inquiète! Pourquoi disent-ils que tu as tué ces deux personnes?

- C'est compliqué ${ }^{100} \ldots "$

Nous observons que le lecteur du roman comprend bien que Sébastian et Nikki sont innocents et qu'ils n'ont pas tué les deux personnes du bar, mais pour ne pas alourdir le texte par des phrases répétées et qui ne font pas progresser l'action du roman, le narrateur ne clarifie pas toutes les informations pour les personnages du roman; le texte progresse et les enquêteurs découvrent la vérité plus tard. Ces points des suspension prouvent que le lecteur est au centre de l'action et l'auteur du roman espère maintenir la tension narrative du lecteur soit par la narration détaillée ou elliptique.

6- Parfois l'emploi des points de suspension traduit la pensée et les sentiments intérieurs des personnages:

-" Je peux tenter de ralentir l'enquête sur les meurtres de Decker, à condition que tu rentres à New York le plus vite possible

$-\ldots$

- D'accod Nikki?

- D'accord, Lorenzo? ${ }^{101 "}$

Les points de suspension expriment la peur de Nikki des enquêtes sur les meurtres et ils traduisent le silence de Nikki vis-à-vis de cette situation épineuse. Les points de suspension remplacent alors une phrase complète de Nikki. L'auteur, par le biais des points des suspensions, essaye de convaincre le lecteur de la netteté de la description et des conversations des personnages.

7- De plus les points de suspension peuvent remplacer un mot grossier ou tabou dans le roman:

"- Ils sont rassis, tes croissants, alors tu vas te sortir les doigts $d u \ldots{ }^{102 "}$.

L'auteur supprime le mot "cul" de l'expression de Constance pour ne pas toucher le lecteur d'un mot tabou, ce qui indique que le lecteur est toujours présent dans l'esprit de l'auteur et qu'il est indissociable ni des paroles des personnages ni des événements narrés du roman.

Bref, les points de suspension sont une manière privilégiée de garantir l'authenticité de la parole, d'apporter au texte une valeur mimétique. Par cette présence massive, Musso rend compte, non 

après... de Guillaume Musso

seulement des paroles de ses personnages, mais également des hésitations, de leurs contrariétés et de leurs repentirs. Cet emploi traduit une pause respiratoire et propose la présence d'un corps qui ne demande qu'à s'exprimer. Dans la parole du narrateur, l'auteur maintient par les points de suspension la tension narrative et il peut aider le lecteur à lui participer l'élaboration de la fiction romanesque. Le lecteur devient alors un partenaire qui découvre le texte et qui peut deviner la pensée des autres, ce qui démontre que l'auteur donne au lecteur le droit d'intervention et de l'échange des idées et des émotions.

\section{Conclusion}

Il nous semble que le roman sept ans après de Musso appartient au type du roman à suspense qui se caractérise par la tension émotive, celle-ci se voit dans la lecture du texte et peut créer aussi une tension narrative du premier degré. Le lecteur reste la plupart du temps inquiet sur l'avenir des personnages et sur l'avenir de l'action dans le roman. Musso excelle à montrer cette tension soit à travers le paratexte soit à travers le texte. Dans le paratexte nous avons observé des choix énigmatiques dans le titre, dans la couverture et dans la quatrième de couverture. Dans le texte, cette tension se traduit dans la voix du narrateur et l'ordre des événements et le choix d'une espace narrative qui convient à la nature des événements dans le roman. Aussi l'adoption des éléments cinématographiques comme le cliffhanger, le flash back et la mise en abyme mettent en évidence cette tension narrative dans le texte, ce qui permet au lecteur d'être encouragé à l'égard de la poursuite des événements du roman. De plus des choix stylistiques et linguistiques peuvent alourdir cette dose du suspense et de la curiosité: emploi massif des points du suspension et des mots étrangers sans accompagnement d' équivalents français, ce qui crée un sentiment de la tension dans la lecture du texte. Bref la lecture du roman policier en général exige une bonne maitrise de ses types pour essayer de délimiter les éléments et la nature des thèmes qui y sont abordés et le roman à suspense en particulier représente un pacte de lecture qui garantit une tension romanesque jusqu'aux derniers chapitres du roman. 
Mohamed Abdelbaki Ahmed

\section{Bibliographie consultée}

\section{1- Corpus}

- Guillaume MUSSO, Sept ans après..., Paris, XO, 2012.

\section{2- Ouvrages consacrés au roman policer et à l'analyse du roman}

- Andréa DEL LUNGO, L'incipit romanesque, Paris, Ed. Seuil, Coll. Poétique, 2003.

- André PEYRONIE, la double enquête du roman policier, Modernités, no 2, 1988.

- Brigitte Moret BUFFARD, Introduction à la stylistique, Paris, Armand Colin, 2005.

- Carole TISSET, Analyse linguistique de la narration, Paris, Ed. Armand Colin, 2000.

- Charles GRIVEL, Production de l'intérêt romanesque, La Haye, Mouton, 1973.

- Daniel FONDANECHE, Le roman policier, Paris, Ellipses, 2000.

- Françoise Rullier-THEURET, le dialogue dans le roman, Paris, Hachette Éducation, 2001.

- Rullier-Theuret Françoise, Approche du roman, Paris, Hachette, 2001

- Gérard GENETTE, $\underline{\text { Seuils, }}$ Paris, édit. du Seuil, 1987.

- Gérard GENETTE, Figures III, Tunisie, Cérès, Coll. CRITICA, 1996.

- George-Elia SARFATI, Eléments d'analyse du discours, Paris, A. Colin, 2007 
La tension narrative dans le roman policier contemporain à travers Sept ans après... de Guillaume Musso

- Jacques BADOU, Jean- Jacques SCHLERET, , Le polar, Paris, Ed. LAROUSSE, 2001.

- Jacques SADOUL, Anthologie de la littérature policière, Paris, Ramsay, 1980.

- Jean DUBOIS, Dictionnaire de linguistique, Paris, Larousse- Bordas, 2002

- Laurence DECREAU, Ces héros qui nous font lire, Paris, Hachette, 1994.

- Leo HOEK, La marque du titre, La Haye, Mouton, 1981.

- Marc LITS, Pour lire le roman policier, Bruxelles, De Boeck, 1989

- Marc LITS, Le genre policier dans tous ses états d'Arsène Lupin à Navarro, Paris, Ed. Pulim, 2011

- Marie-Luce GION, Pierrette SLAMA, lire et écrire avec le roman policier, Lille, CRDP de l'Académie Créteil, 1998.

- Maurice DELCROIX, Fernand HALLYN, Introduction aux études littéraires: méthodes du texte, Paris, Duclot, 1995.

- Michèle PERRET, L'énonciation en grammaire du texte, Paris, Nathan, 1997.

- Michel POUGEOISE, Dictionnaire didactique de la langue francaise, Paris, Armand Collin, 1996.

Muriel ROSEMBERG, Le roman policier, lieux et itinéraires, Paris, Ed. L'Harmattan, 2007,

- Raphael BARONI, la valeur littéraire du suspense, A contrario, no.1, 2004 
- Raphaël BARONI, La tension narrative. Suspense, curiosité et surprise, Paris, Éditions du Seuil, 2007.

- Raphaël Baroni, «Le cliffhanger : un révélateur des fonctions du récit mimétique », Cahiers de Narratologie [En ligne], $31 \mid 2016$,consulté le 10 septembre 2018. URL : http://journals.openedition.org/narratologie/7570

- Roland BOURNEUF, Réal OUELLET, L'univers du roman, Paris, Ed. PUF, $5^{\text {ième }}$ édition, 1989.

- Vincent JOUVE, La poétique du roman, Paris, Ed. Armand Colin, 2001

- Yves REUTER, Le roman policier,_Paris, Nathan, 1999

- Yves REUTER, Introduction à l'analyse du roman, Paris, Ed. Armand Colin, 2009.

\section{Notes:}

${ }^{1}$ Cf., Jacques SADOUL, Anthologie de la littérature policière, , Paris, Ramsay, 1980, p. 10.

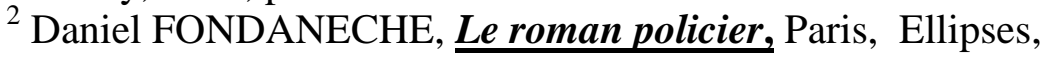
2000.,p.14

${ }^{3}$ Edgar Poe a écrit une trilogie: Le double assassinat dans la rue Morgue en 1841, la lettre volée en 1842, et le Mystère de Marie Roget en 1843. Nous pouvons citer Arthur Canon Doyle qui a créé dans ses romans publiés de 1887 à 1927 le prototype du détective privé Sherlock Holmes. Dans la lignée, Agatha Christie avec Hercule Poirot réalise un succès immense. L'école française du genre policier a ses grands noms comme Maurice Leblanc avec son justicier Arsène Lupin et Gaston Leroux avec le détective Rouletabille. Ces textes ont servi de modèle à des séries futures comme Le club des cinq ou Alice. Vient ensuite les romans de Lorris Murail qui met en scène le jeune Dan Martin. Les héros des romans policiers ne sont pas considérés des hommes ordinaires mais des surhommes, puisque les détectives reviennent d'un récit à un autre afin de rétablir l'ordre et la justice.

${ }^{4}$ Laurence DECREAU: Ces héros qui nous font lire, Paris, Hachette, 1994, P.23 
La tension narrative dans le roman policier contemporain à travers Sept ans après... de Guillaume Musso

${ }^{5}$ Cf., André PEYRONIE, La double enquête du roman policier, Modernités ,no 2, 1988, p.129.

6 Jacques BADOU, Jean- Jacques SCHLERET, Le polar, Paris, Ed. Larousse, 2001,.P.6.

${ }^{7}$ Marc LITS, Pour lire le roman policier, Bruxelles, De Boeck, 1989, P.86.

${ }^{8}$ Muriel ROSEMBERG, Le roman policier, lieux et itinéraires, Paris, Ed.

L'Harmattan, 2007, P.3.

${ }^{9}$ Marc LITS, Le genre policier dans tous ses états d'Arsène Lupin à Navarro, Paris, Ed. Pulim, 2011, P.15.

${ }^{10}$ Ibid, pp:63:64.

${ }^{11}$ Jacques BADOU, Jean- Jacques SCHLERET, $\underline{\boldsymbol{p} \text { p.-cit., P.6. }}$

${ }_{12}$ Cf, Yves REUTER, Le roman policier, Paris, Nathan, 1999., p.75

${ }^{13}$ Raphael BARONI, la valeur littéraire du suspense, A contrario, no.1, 2004, p.41.

${ }^{14}$ L'action du roman se déroule autour Sébastian et Nikki qui sont séparés depuis des années et ont chacun la garde de l'un de leurs jumeaux, Camille et Jeremy. Mais la disparition de Jeremy les obligent tous deux à se sacrifier une nouvelle fois, à s'entendre et à s'organiser pour essayer de le retrouver. Ils sont loin d'imaginer que le complexe jeu de piste qu'ils suivent jusqu'à Paris a été planifié par leurs enfants pour les voir à nouveau réunis dans la même maison. Mais dès le départ, les choses se compliquent, car Camille et Jeremy deviennent la cible d'un dangereux cartel brésilien qui cherche une cargaison de cocaïne, perdue dans un crash d'un avion dans la jungle. Tous les deux s'efforcent de retrouver leurs enfants, à l'aide de deux policiers, et ils retrouvent aussi des sentiments qu'ils croyaient perdus à jamais.

${ }^{15} \mathrm{Cf}$., Marie-Luce GION, Pierrette SLAMA, lire et écrire avec le roman policier, Lille, CRDP de l'Académie Créteil, 1998, P.51,52.

${ }^{16}$ Leo HOEK, La marque du titre , La Haye, Mouton, 1981, P.17.

${ }^{17}$ Charles GRIVEL, Production de l'intérêt romanesque, La Haye, Mouton, 1973, P.166.

${ }^{18}$ Jean DUBOIS, Dictionnaire de linguistique, Paris, Larousse- Bordas, 2002, p.459

${ }^{19}$ Raphael BARONI, La valeur littéraire du suspense, Op.Cit., P.30.

20 Cf., Marie-Luce GION, Pierrette SLAMA, $\underline{O p}$. Cit ., P.56

21 Nous pouvons rencontrer certains éditeurs qui refusent tout guidage de lecture et présentent des quatrièmes totalement vierges comme dans la collection blanche de Gallimard. Selon ces éditeurs, la garantie de qualité réside essentiellement dans le nom de l'auteur et la notoriété de la maison d'édition qui publie l'ouvrage.

${ }^{22}$ Raphael BARONI, La valeur littéraire du suspense, Op.Cit.,p. 31. 
${ }^{23}$ Cf., Marie-Luce GION, Pierrette SLAMA, Op. Cit.P.58.

24 Andréa DEL LUNGO, L'incipit romanesque, Paris, Ed. Seuil, Coll. Poétique, , 2003., P.14.

25 Yves REUTER, Introduction à l'analyse du roman, Paris, Ed. Armand Colin, 2009, P.141.

${ }^{26}$ Gerard GENETTE, Seuils, Op.-cit., P.21

${ }^{27}$ Ibid., P.22

28 Guillaume MUSSO, Sept ans après..., , Paris, XO, 2012., P.9.

${ }^{29}$ Ibid., P.10

30 Ibid, P.11

$31 \frac{\text { Ibid. }}{\text {. P.13 }}$

32 Il y a d'autres fonctions de l'incipit comme la fonction dramatique, et la fonction codifiante. Cf., Vincent JOUVE, La poétique du roman, Paris, Ed. Armand Colin, 2001, P.37.

33 CF. Marie-Luce GION, Pierrette SLAMA, Op. Cit., P.58

${ }^{34}$ Gérard GENETTE, Seuils, Op. Cit., P.376.

${ }^{35}$ Raphaël BARONI, La tension narrative. Suspense, curiosité et surprise, Paris, Éditions du Seuil, 2007. p.128.

36 Gérard GENETTE, Figures III, Cérès, Tunisie, Coll.CRITICA, 1996. PP:252,253.

37 G.MUSSO, Sept ans après..., P.21

38 Ibid., 31

39 Reuter Yves, L'analyse du récit, Op.-cit., P.43

${ }^{40}$ Carole TISSET, Analyse linguistique de la narration, Paris, Ed. Armand Colin, 2000, P.90.

${ }^{41}$ G.MUSSO, Sept ans après..., P.181

${ }^{42}$ Ibid., P.159

43 Gerorge-Elia SARFATI, Eléments d'analyse du discours, Paris, A.Colin, 2007, P.64.

${ }^{44}$ G.MUSSO, Sept ans après..., P.123

${ }^{45}$ Ibid, P.188

46 Ibid., P.206

47 Michèle PERRET, L'énonciation en grammaire du texte, Paris, Nathan, 1997, P.101

${ }^{48}$ Francoise Rullier-THEURET, le dialogue dans le roman, Paris, Hachette Éducation, 2001, p.76.

${ }^{49}$ G.MUSSO, Sept ans après..., P.31

${ }^{50}$ Francoise Rullier-THEURET, Op.Cit., , p.75

${ }^{51}$ G.MUSSO, Sept ans après..., P.145.

${ }^{52}$ Ibid., P.27

53 Ibid., P.26 
La tension narrative dans le roman policier contemporain à travers Sept ans après... de Guillaume Musso

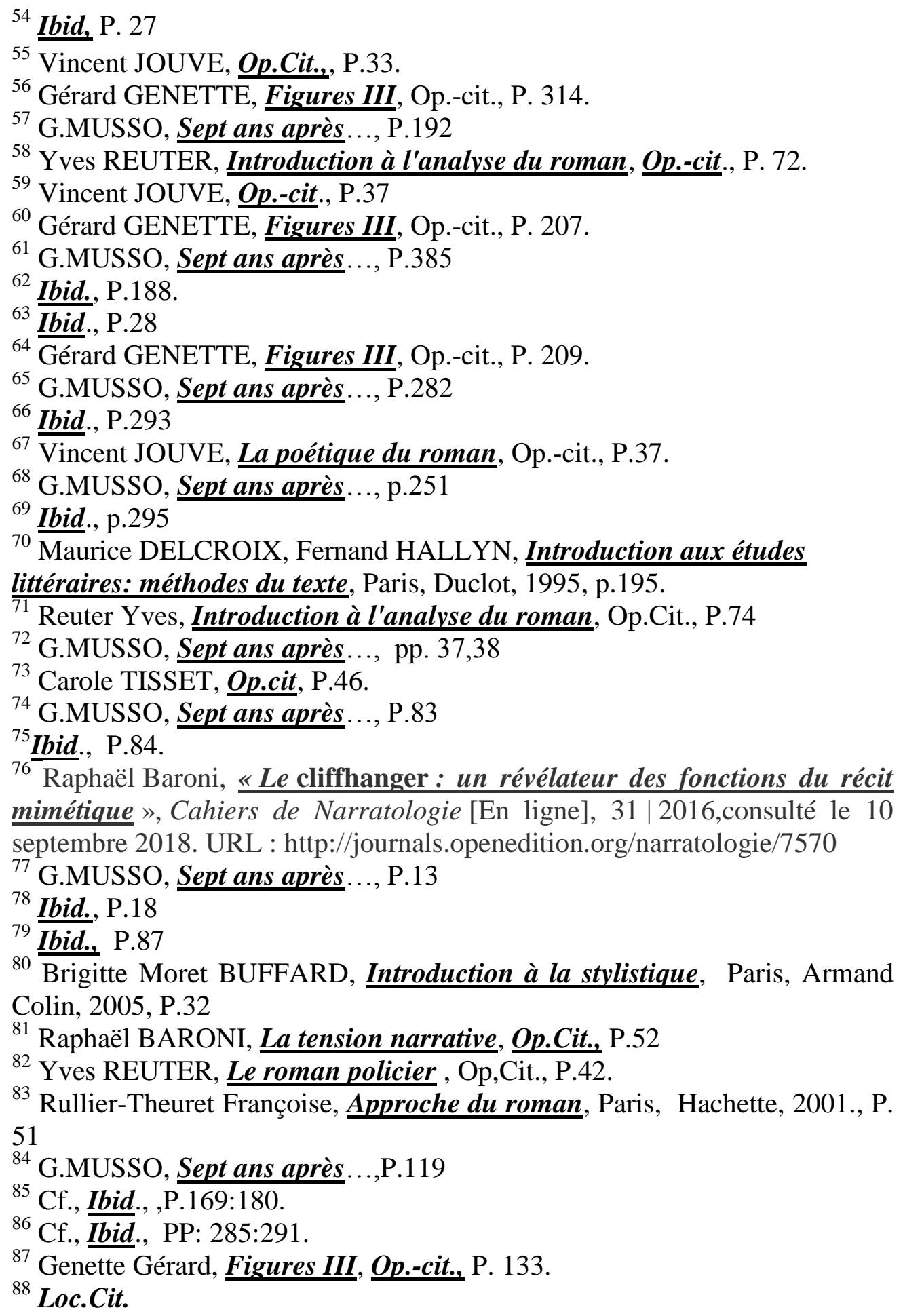


${ }^{89}$ G.MUSSO, Sept ans après..., P.362.

${ }^{90}$ Ibid., P.323

$91 \frac{\text { Ibid., }}{\text { Ib.15 }}$

92 Ibid., PP:148:149

$93 \frac{\text { Ibid., P.322 }}{\text { Ibid. }}$

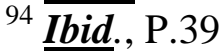

95 Ibid., P. 56

96 Ibid., P.69

97 Loc.Cit

98 Ibid., PP: 80,81.

99 Ibid., P.263

${ }^{100}$ Ibid. P.246

${ }^{101}$ Ibid.,P.165

${ }_{102}$ Ibid, p.265 